\title{
ST2 and Multimarker Testing in Acute Decompensated Heart Failure
}

\author{
Alexandre Mebazaa, MD ${ }^{\mathrm{a}} *$, Salvatore Di Somma, MD ${ }^{\mathrm{b}}$, Alan S. Maisel, MD ${ }^{\mathrm{c}}$, \\ and Antoni Bayes-Genis, MD ${ }^{\mathrm{d}, \mathrm{e}}$
}

\begin{abstract}
Most data on heart failure biomarkers have been derived from patient cohorts with chronic disease. However, risk prediction in patients admitted with acute decompensated heart failure (ADHF) remains a challenge. ADHF is not a single disease: it presents in various manners, and different causes may underlie ADHF, which may be reflected by different biomarkers. Soluble suppression of tumorigenicity 2 (ST2) has been shown to be a strong independent predictor of short-, mid-, and long-term outcome in ADHF. Furthermore, combining biomarkers may help further improve the prognostic power of ST2. The ProBNP Investigation of Dyspnea in the Emergency Department study showed that elevated plasma levels of ST2 together with elevated levels of 4 other biomarkers have clear incremental values to predict outcome in ADHF. The Multinational Observational Cohort on Acute Heart Failure study is an international collaborative network that recruited 5,306 patients hospitalized for ADHF that demonstrated that ST2 and midregional pro-adrenomedulin had independently strong value to predict 30-day and 1-year outcome in patients with ADHF. The Multinational Observational Cohort on Acute Heart Failure study also showed that C-reactive protein plus ST2 better classified risk in patients with ADHFs than ST2 alone. Combining biomarkers for risk prediction or risk stratification might have clinical and more importantly pathophysiological meaning. () 2015 Elsevier Inc. All rights reserved. (Am J Cardiol 2015;115[suppl]:38B-43B)
\end{abstract}

The use of biomarkers to assist in the care of acute decompensated heart failure (ADHF) patients has been an area of vastly expanding research. Natriuretic peptides (NPs) represent the current biomarker gold standard for diagnosis and prognosis in patients with ADHF. ${ }^{1}$ Subsequent study of the NPs demonstrates considerable opportunity to add to the prognostic information gained from their measurement. In this regard, newer biomarkers have been studied either alone or together with the NPs. Elevated plasma levels of the interleukin receptor family member suppression of tumorigenicity 2 (ST2) ${ }^{2}$ and others, such as midregional pro-adrenomedullin (MR-proADM), the pro-peptide of a prognostically meaningful endogenous vasoconstrictor, ${ }^{3}$ have been individually shown to be associated with poorer outcome in ADHF.

\footnotetext{
${ }^{a}$ Université Paris Diderot, PRES Sorbonne Paris Cité and Saint Louis Lariboisière University Hospitals, Paris, France; ${ }^{b}$ Department of MedicalSurgery Sciences and Translational Medicine, University Sapienza Rome, Sant' Andrea Hospital, Rome, Italy; ${ }^{\mathrm{c}}$ Department of Medicine, VA San Diego Healthcare System, San Diego, California; ${ }^{\mathrm{d} C a r d i o l o g y}$ Service, Hospital Universitari Germans Trias i Pujol, Barcelona, Spain; and ${ }^{\mathrm{e} D e-}$ partment of Medicine, Universitat Autonoima de Barcelona, Barcelona, Spain.

Publication of this supplement was supported by funding from Critical Diagnostics, San Diego, CA.

Statement of author disclosure: Please see the Author Disclosures section at end of this article.

*Address for reprints: Alexandre Mebazaa, MD, Université Paris Diderot, PRES Sorbonne Paris Cité and Saint Louis Lariboisière University Hospitals, U942 Inserm, Paris, France.

E-mail address: alexandre.mebazaa@1rb.aphp.fr.
}

Recently, various studies tested combined biomarkers to predict outcome in ADHF. Indeed, plasma levels of NPs are more related to myocardial stress, whereas MR-proADM might be more related to global stress and ST2 might better reflect ventricular fibrosis and remodeling. Combining those novel biomarkers was shown to have additive values.

\section{Clinical Models to Risk Stratify Severe Critically III Patients}

Different prognostic systems, such as the Acute Physiology and Chronic Health Evaluation II or III, the Simplified Acute Physiology Score (SAPS) II, and the Mortality Probability Models II, have been developed to predict the outcome of critically ill patients admitted to the intensive care unit (ICU). SAPS II provides a method to convert the score to a probability of hospital mortality ${ }^{4}$ and includes several key clinical parameters and biological markers, measured all at admission and known to alter outcome. Clinical parameters include age, gender, cause of ICU admission, co-morbidities, blood pressure, heart rate, and temperature. Several biological parameters recorded at admission are used to calculate the SAPS II, including arterial oxygenation, bicarbonates, liver and renal function markers, and potassium and sodium. SAPS II score can, however, only be measured at admission and is not used to follow patient condition. SAPS II has been shown to be a moderate marker of 28-day outcome.

To follow the severity of patients admitted in the ICU during their initial hospital stay, an organ dysfunction score 

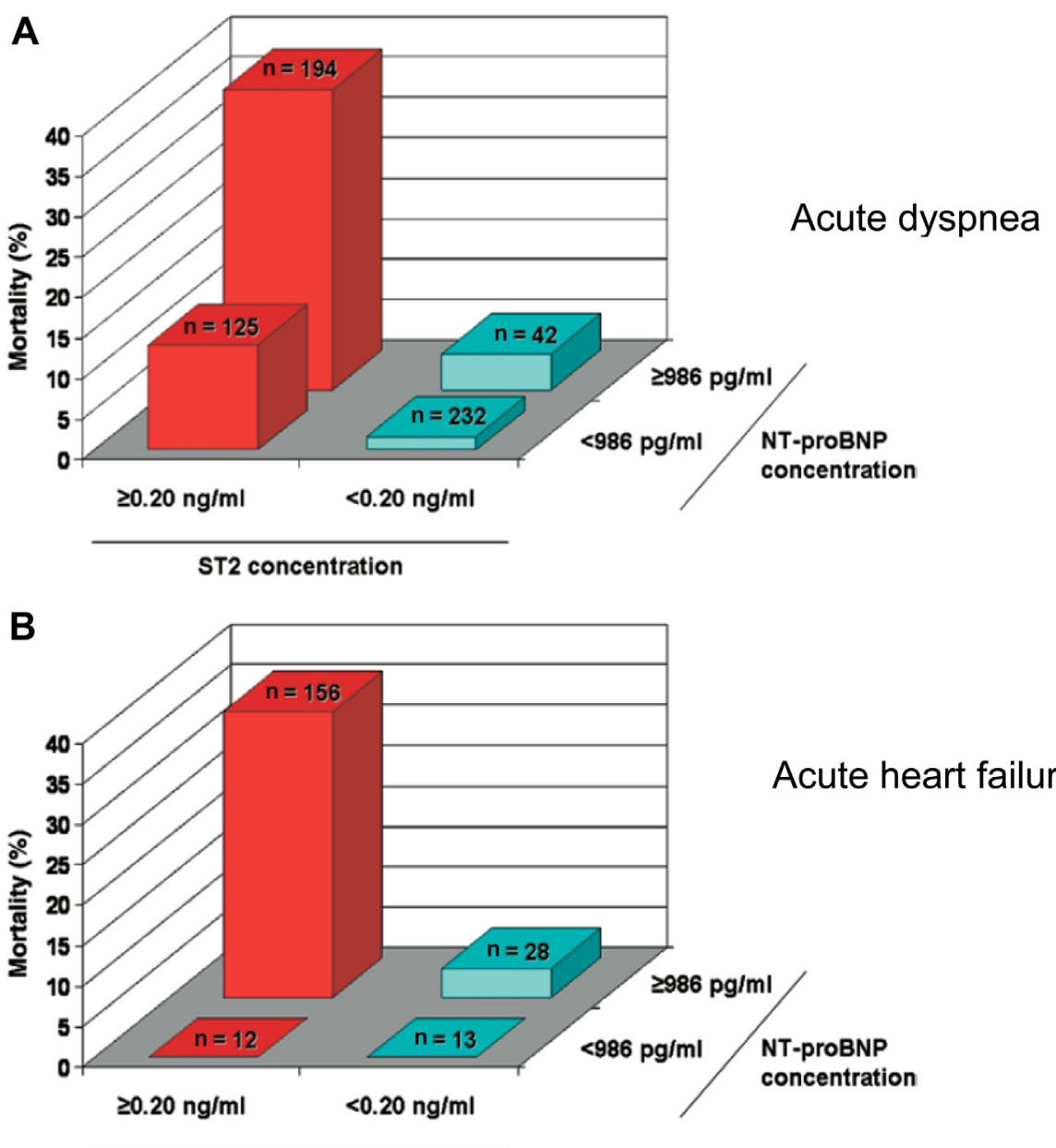

ST2 concentration

Figure 1. Mortality rates at 1 year as a function of ST2 and NT-proBNP concentrations. Concentrations of sST2 were measured using an early research-useonly assay rather than the Presage ST2 method. From Januzzi et $\mathrm{al}^{2}$ with permission.

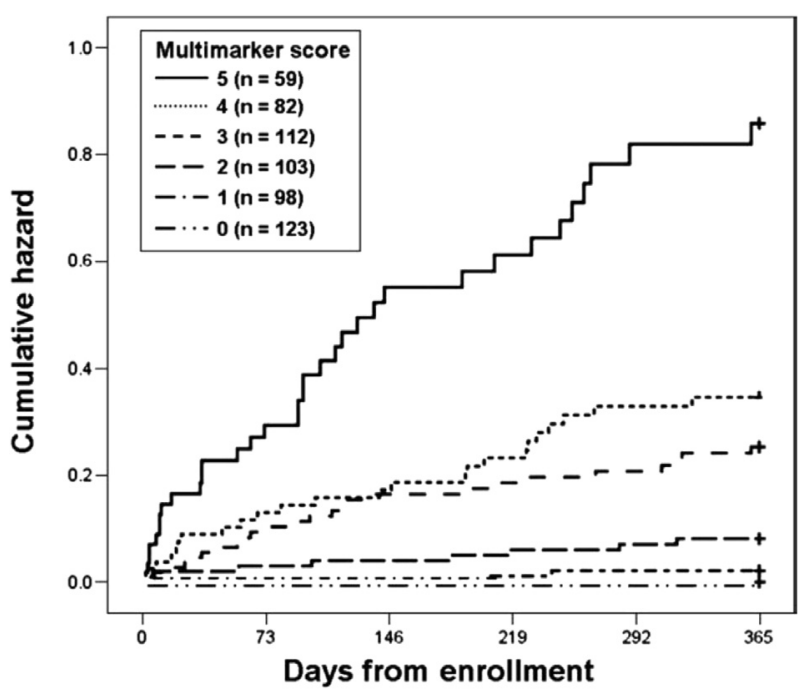

Figure 2. Cumulative hazard curves demonstrating the ability of a multimarker strategy using CRP, ST2, BUN, hemoglobin, and NT-proBNP to predict hazard both early and in a sustained fashion. From Rehman et al ${ }^{8}$ with permission. $\mathrm{BUN}=$ blood urea nitrogen. has been developed, The Sepsis-Related Organ Failure Assessment (SOFA) score to describe organ dysfunction/ failure. ${ }^{5}$ The SOFA score is mostly based on biological markers and has been seen by intensivists as a "multimarker strategy" to predict outcome. Indeed, in septic patients, various organ dysfunctions are present at admission, and the degree and the number of organ dysfunction are associated with outcome. SOFA score was described to assess the degree and the number of organ dysfunction at admission and during the following days. SOFA score is mostly based on biological markers and includes respiratory, kidney, liver, and coagulation markers. Importantly, none of the aforementioned scores include measurement of cardiac biomarkers.

\section{Combined Troponin and NPs Use in ADHF}

Plasma measurements of troponin and NPs are recommended in the initial phase of ADHF management. Plasma troponin levels may help ruling out an acute myocardial infarction as a main cause of ADHF. Elevated cardiac troponin levels at 
Table 1

Risk prediction and reclassification for all-cause one-year mortality in ADHF with a combination of plasma CRP and ST2 at admission to a clinical model

\begin{tabular}{|c|c|c|c|c|c|}
\hline \multirow[t]{2}{*}{ Alive } & \multirow[t]{2}{*}{ Predicted mortality } & \multicolumn{4}{|c|}{ Clinical + biomarker $(\mathrm{CRP}+\mathrm{sST} 2)$ model } \\
\hline & & Low $<20 \%$ & Intermediate $20-60 \%$ & High $>60 \%$ & Total \\
\hline \multirow[t]{4}{*}{ Clinical model } & Low $<20 \%$ & 121 & 15 & $\mathbf{0}$ & 136 \\
\hline & Intermediate $20-60 \%$ & 50 & 111 & 13 & 174 \\
\hline & High $>60 \%$ & $\mathbf{0}$ & 6 & 4 & 10 \\
\hline & Total & 171 & 132 & 17 & 320 \\
\hline \multirow[t]{2}{*}{ Dead } & Predicted mortality & \multicolumn{4}{|c|}{ Clinical + biomarker $(\mathrm{CRP}+\mathrm{sST} 2)$ model } \\
\hline & & Low $<20 \%$ & Intermediate $20-60 \%$ & High $>60 \%$ & Total \\
\hline \multirow[t]{4}{*}{ Clinical model } & Low $<20 \%$ & 14 & 5 & $\mathbf{0}$ & 19 \\
\hline & Intermediate 20 - $60 \%$ & 8 & 73 & 22 & 103 \\
\hline & High $>60 \%$ & $\mathbf{0}$ & 3 & 13 & 16 \\
\hline & Total & 22 & 81 & 35 & 138 \\
\hline
\end{tabular}

NRI [95\%CI]: 20.3[ 9.9-30.7], p-value: $<0.001$; NRI alive $8.8 \%$; NRI dead $11.6 \%$.

IDI [95\% CI]: 0.08[ 0.05-0.10]; p-value: $<0.001$.

Comparing the clinical model versus the clinical+biomarker (CRP+ST2) model, the green cells are patients who changed risk category in a beneficial direction with addition of biomarkers to the model, while light blue numbers are patients who were reclassified in the inappropriate direction. Patients in the diagonal boxes ( grey) have the same predicted risk with both models. The top table concerns one-year survivors and bottom table one-year non-survivors. From the top table, the NRI alive is calculated as follows: $(56-26) / 320=0.88$ (or $8.8 \%$ ). From $\mathrm{J}_{\text {Lassus et al }}{ }^{12}$ with permission.

admission have also been correlated with poor prognosis. It has further been demonstrated that patients with ADHF with detectable troponin I and elevated BNP were at particularly high risk of death, whereas patients with ADHF without detectable troponin I and lower BNP levels had a substantially lower risk of adverse outcome. ${ }^{6}$ With the development and spread of highly sensitive troponin methods, the prognostic impact of troponin measurement has increased.

\section{Multimarker Strategy Including ST2 in Patients Admitted for Acute Dyspnea}

Pascual-Figal et $\mathrm{al}^{7}$ prospectively studied 107 patients hospitalized with ADHF (mean age $72 \pm 13$ years, $44 \%$ men, left ventricular ejection fraction $47 \pm 15 \%$ ). Blood samples were collected on presentation to measure ST2 (reflective of myocardial fibrosis and remodeling), high-sensitivity troponin $\mathrm{T}$ (hsTnT-indicative of myocardial necrosis), and N-Terminal Pro-B-Type Natriuretic Peptide (NT-proBNP) (identifying myocardial stretch) levels. Each provided independent and additive prognostic information for death in the shorter and longer term. Furthermore, a simple combined multimarker score powerfully identified patients at low, intermediate, and high risk for all-cause mortality. The presence of none, 1,2, or 3 elevated biomarkers was associated with a strikingly steeply rising incremental risk of death, ranging from $0 \%$ to $50 \%$. The addition of this simple multimarker score significantly increased the predictive performance achieved by the entire model taking into account clinical predictors plus NT-proBNP levels. The survival curves suggest that biomarkers add prognostic information during the first year and clearly separate those patients at low risk (none or 1 biomarker elevated) from those at high risk ( $\geq 2$ biomarkers elevated). This simple and objective ability to stratify patients into very low and very high risk could be used to triage patients with ADHF to different therapeutic interventions, ranging from low-risk HF observation units to earlier triage to mechanical support for those at very high risk.

A multimarker strategy has also been evaluated by the Pro-B-type natriuretic peptide Investigation of Dyspnea in the Emergency Department (PRIDE) Investigators. ${ }^{2}$ Of 560 patients from PRIDE, 180 had ADHF. Concentrations of NT-proBNP, MR-proADM, and MR-proANP were measured, and patients were followed for 4 years for survival. In comprehensive statistical analyses, both midregional peptides were associated with death out to 4 years, individually or in a multimarker strategy. Figure 1 further shows that the association of ST2 and NT-proBNP might have additive values to predict 1-year outcome in patients with both acute heart failure and more globally acute dyspnea.

Using the same PRIDE database, in addition to ST2 and NT-proBNP, other plasma markers might have additional values (87) (Figure 2). Thus, elevated values of ST2, NT-proBNP, C-reactive protein (CRP), hemoglobin, and blood urea nitrogen provided independent and incremental prognostic information. The number of increased biological markers, assessed simultaneously after admission for acute dyspnea, provided powerful prognostic information that was independent and additive to each other.

\section{Novel Statistical Methods to Assess an Improved Performance of Biomarkers}

Net reclassification improvement (NRI) has recently gained attention for examining value of biomarkers in clinical care. Traditionally, standard prognostic models assessed incremental value of biomarkers by comparing the area under the curve of the receiver operating characteristic between a 

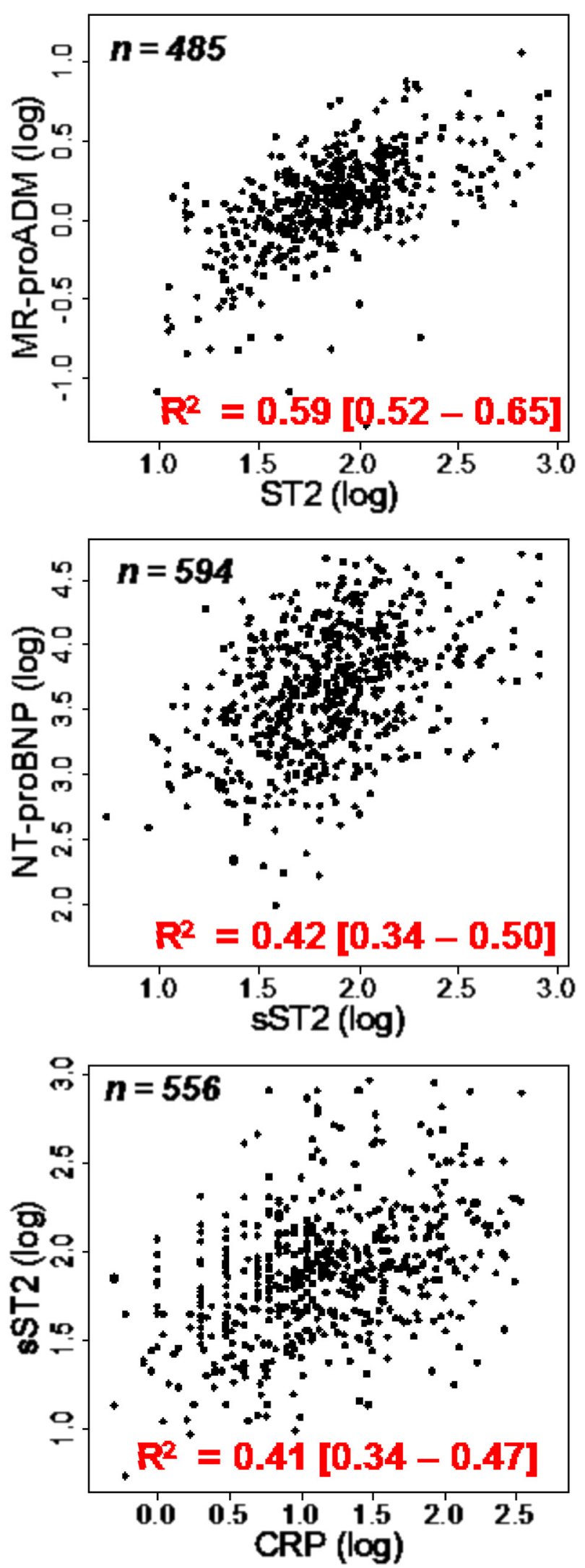

Figure 3. Correlations between ST2 and various cardiovascular biomarkers in patients with $\mathrm{ADHF}$. clinical model alone versus a clinical model + biomarkers. Discrimination (by means of area under the curve or $c$-statistic) achieved popularity in diagnostic testing because sensitivity and specificity test characteristics are relevant to elucidate diseased versus nondiseased patients. Discrimination alone was extensively used in pioneer articles on NPs ${ }^{9}$ and ST2 in patients admitted with acute dyspnea. ${ }^{2}$ However, discrimination by itself may not be optimal when comparing models for risk prediction or to stratify individuals into risk categories. ${ }^{10}$

More recently, Pencina et $\mathrm{al}^{11}$ introduced a distinction between risk "prediction" and risk "classification," suggesting that measures such as patient reclassification, which goes beyond statistical significance, and $c$-statistic are necessary. Thus, as listed in Table 1, NRI offers a simple intuitive way of quantifying improvement offered by novel markers on the top of clinical model. Studies focused on NRI yielded by ST2 demonstrate favorable data in ADHF, with additive value compared with other biomarkers, for example, the combination of CRP plus ST2 markedly improved risk stratification in patients with ADHF (Table 1). Such improvement in risk stratification was seen both in survivors and nonsurvivors of ADHF. ${ }^{7}$ Among decedents, the NRI of patients dying was $11.6 \%$. This meant that at least $11.6 \%$ of the patients with ADHF were better classified into higher risk using CRP + ST2 on top of the clinical model than the clinical model alone. Among survivors, the NRI for survivors was $8.8 \%$.

\section{Plasma-Soluble ST2 Alone or Combined to Other Biomarkers for Risk Stratification of Patients With ADHF}

We compared various novel and known prognostic cardiovascular biomarkers reflective of different pathobiological pathways to predict outcome in a large cohort of patients with ADHF in The Multinational Observational Cohort on Acute Heart Failure study. ${ }^{12}$ To explore whether ST2 might be correlated to other prognostic biomarkers, we first compared plasma concentration of ST2, NT-proBNP, MR-proADM, and CRP. ${ }^{12}$ Figure 3 shows a relatively strong correlation observed between MR-proADM and ST2 $(\mathrm{R}=0.59$, $\mathrm{p}<0.001$ ), with moderate correlations found between NT-proBNP and ST2 $(\mathrm{R}=0.42, \mathrm{p}<0.001)$. CRP showed weak associations with ST2.

The Multinational Observational Cohort on Acute Heart Failure study was designed to equitably assess the individual and added value of various novel biomarkers (including ST2) to traditional clinical variables. Figure 4 illustrates that both ST2 and MR-proADM had striking NRI beyond clinical variables to predict 30-day mortality. This demonstrates that if measured at admission, each of the 2 novel biomarkers markedly improve the risk stratification of patients with ADHF. Although combination of ST2 with other biomarkers, such as CRP, did not add much to predict 30-day 
A 30-day mortality

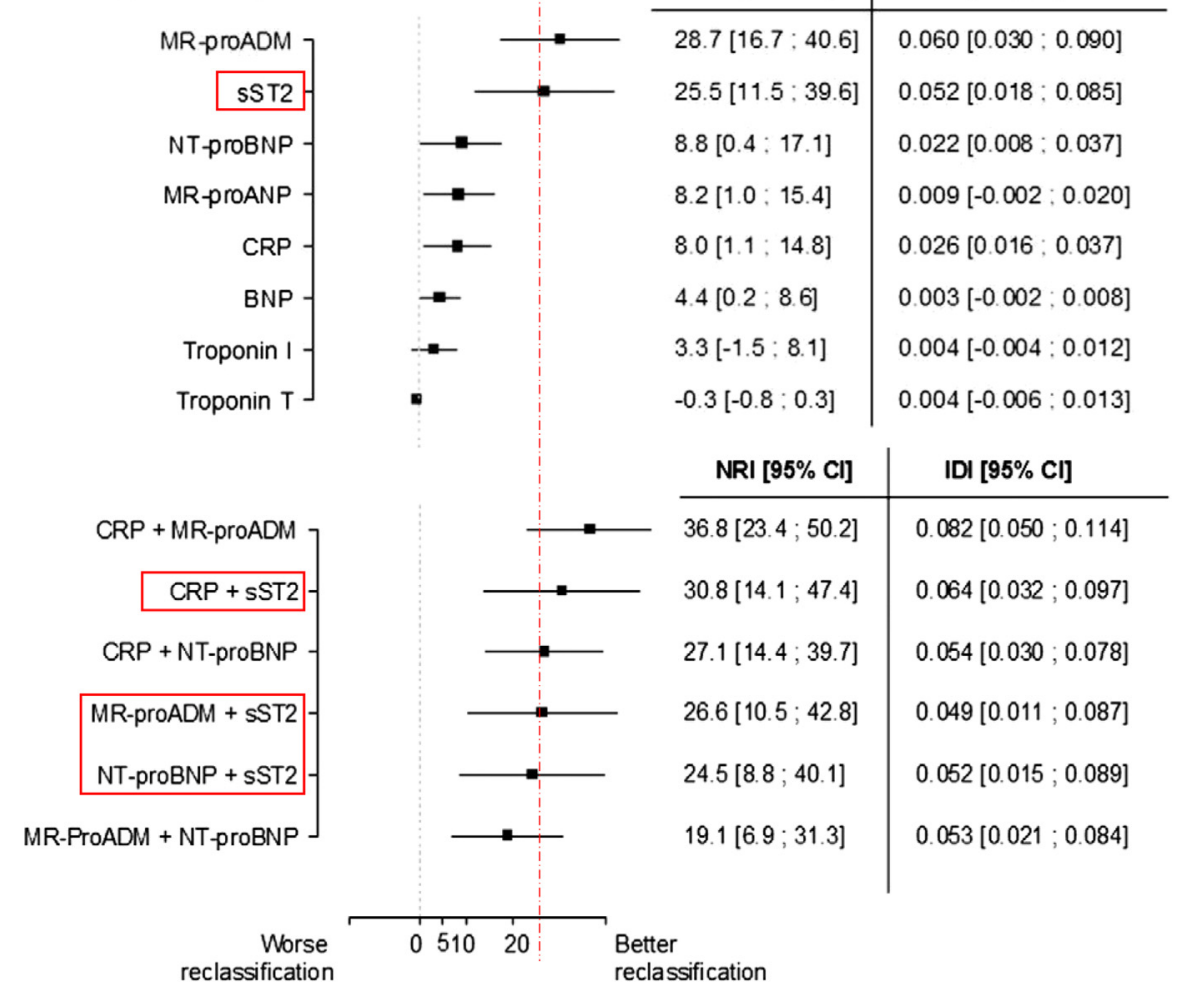

Acute dyspnea $n=593$

\section{B One-vear mortality}

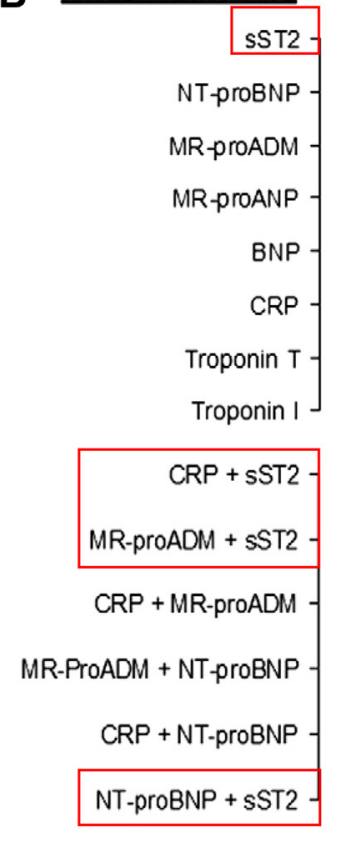

The American Journal of Cardiology (www.AJConline.org) Vol 115 (7S) April 2, 2015

\begin{tabular}{c|c} 
NRI [95\% CI] & IDI [95\% CI] \\
\hline $28.7[16.7 ; 40.6]$ & $0.060[0.030 ; 0.090]$ \\
$25.5[11.5 ; 39.6]$ & $0.052[0.018 ; 0.085]$ \\
$8.8[0.4 ; 17.1]$ & $0.022[0.008 ; 0.037]$ \\
$8.2[1.0 ; 15.4]$ & $0.009[-0.002 ; 0.020]$ \\
$8.0[1.1 ; 14.8]$ & $0.026[0.016 ; 0.037]$ \\
$4.4[0.2 ; 8.6]$ & $0.003[-0.002 ; 0.008]$ \\
$3.3[-1.5 ; 8.1]$ & $0.004[-0.004 ; 0.012]$ \\
$-0.3[-0.8 ; 0.3]$ & $0.004[-0.006 ; 0.013]$ \\
NRI [95\% Cl] & IDI [95\% Cl] \\
\hline $36.8[23.4 ; 50.2]$ & $0.082[0.050 ; 0.114]$ \\
$30.8[14.1 ; 47.4]$ & $0.064[0.032 ; 0.097]$ \\
$27.1[14.4 ; 39.7]$ & $0.054[0.030 ; 0.078]$ \\
$26.6[10.5 ; 42.8]$ & $0.049[0.011 ; 0.087]$ \\
$24.5[8.8 ; 40.1]$ & $0.052[0.015 ; 0.089]$ \\
$19.1[6.9 ; 31.3]$ & $0.053[0.021 ; 0.084]$ \\
&
\end{tabular}

\begin{tabular}{l|c} 
NRI [95\% CI] & IDI [95\% CI] \\
\hline $10.3[1.9 ; 18.7]$ & $0.048[0.028 ; 0.067]$ \\
$9.1[4.0 ; 14.1]$ & $0.025[0.016 ; 0.034]$ \\
$9.1[2.4 ; 15.8]$ & $0.042[0.028 ; 0.057]$ \\
$7.4[1.6 ; 13.2]$ & $0.028[0.014 ; 0.041]$ \\
$5.5[1.5 ; 9.4]$ & $0.020[0.012 ; 0.027]$ \\
$5.3[1.9 ; 8.8]$ & $0.011[0.005 ; 0.016]$ \\
$0.0[-0.9 ; 1.0]$ & $0.000[-0.001 ; 0.002]$ \\
$-0.2[-1.8 ; 1.5]$ & $0.000[-0.001 ; 0.002]$ \\
$20.3[9.9 ; 30.7]$ & $0.076[0.050 ; 0.102]$ \\
$16.2[6.2 ; 26.2]$ & $0.060[0.037 ; 0.083]$ \\
$15.3[7.9 ; 22.7]$ & $0.055[0.038 ; 0.072]$ \\
$14.6[7.8 ; 22.2]$ & $0.052[0.035 ; 0.069]$ \\
$13.8[7.4 ; 20.1]$ & $0.039[0.025 ; 0.052]$ \\
$12.4[3.6 ; 21.3]$ & $0.055[0.035 ; 0.076]$ \\
&
\end{tabular}

Acute heart failure $n=208$

Figure 4. Cumulative hazard curves demonstrating the ability of a multimarker strategy using CRP, ST2, BUN, hemoglobin, and NT-proBNP to predict hazard both

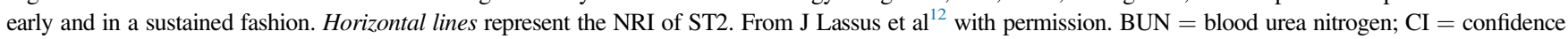
interval. 


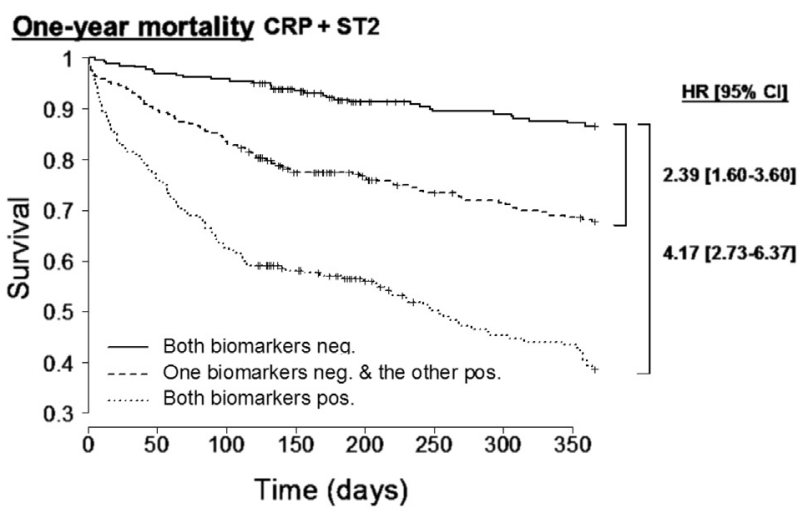

Figure 5. Kaplan-Meier of survival rate comparing various CRP + ST2 levels. $\mathrm{CI}=$ confidence interval; $\mathrm{HR}=$ hazard ratio; neg. = negative; pos. $=$ positive.

mortality, it had important effect of 1-year mortality. KaplanMeier curves (Figure 5) confirmed a twofold increased risk of death in patients with ADHF with elevated plasma levels of ST2 or CRP and fourfold increased risk if both CRP and ST2 were elevated.

\section{Future Perspectives and Unanswered Questions}

ADHF is a complex cardiovascular disease with alteration of various organ functions making analysis of clinical and "classical" biological tests complex to interpret. A better understanding of the best combination of biomarkers for evaluating risk in a multimarker strategy is needed. Furthermore, identifying therapeutic strategies that might follow recognition of an elevated set of such biomarkers is a much needed next step.

In conclusion, novel biomarkers, either alone or in combination as multimarker panels, have been shown to markedly improve and clarify the prognosis of ADHF. Among them, ST2 emerges as a candidate biomarker to be incorporated in such panels given its relevant impact in discrimination and patient reclassification. Future new biomarkers may eventually provide additive values to risk stratification of patients with ADHF to optimize management in both the initial phase at admission and later during the hospital stay.

\section{Author Disclosures}

Alexandre Mebazaa reports receiving speaker's honoraria from and/or was member of advisory board of Alere, Thermo Fisher, Cardiorentis, Vifor, Edwards, Novartis, Critical Diagnostics, Orion, and Bayer. Salvatore Di Somma reports receiving travel support, speakers fees, research grants, and/or consultancy fees from BG Medicine, Biomerieux, Alere, Adrenomed, Sphingotec, Thermo Fisher, Critical Diagnostics, Cardiorentis, and Novartis. Alan S. Maisel reports receiving grant support from Alere, Abbott, BG Medicine, and Thermo Fisher; consulting fees from
Alere, Critical Diagnostics, Sphingotec, BG Medicine, and EFG Diagnostics; speaking fees from BG Medicine and Alere; and ownership interest in Cardero Therapeutics and My Life Diagnostics. Antoni Bayes-Genis reports receiving grant support from Roche Diagnostics and consulting income from Critical Diagnostics and Roche Diagnostics.

1. Cohen-Solal A, Logeart D, Huang B, Cai D, Nieminen MS, Mebazaa A. Lowered B-type natriuretic peptide in response to levosimendan or dobutamine treatment is associated with improved survival in patients with severe acutely decompensated heart failure. J Am Coll Cardiol 2009;53:2343-2348

2. Januzzi JL Jr, Peacock WF, Maisel AS, Chae CU, Jesse RL, Baggish AL, O'Donoghue M, Sakhuja R, Chen AA, van Kimmenade RR, Lewandrowski KB, Lloyd-Jones DM, Wu AH. Measurement of the interleukin family member ST2 in patients with acute dyspnea: results from the PRIDE (Pro-Brain Natriuretic Peptide Investigation of Dyspnea in the Emergency Department) study. J Am Coll Cardiol 2007;50:607-613.

3. Maisel A, Mueller C, Nowak R, Peacock WF, Landsberg JW, Ponikowski P, Mockel M, Hogan C, Wu AH, Richards M, Clopton P, Filippatos GS, Di Somma S, Anand I, Ng L, Daniels LB, Neath SX, Christenson R, Potocki M, McCord J, Terracciano G, Kremastinos D, Hartmann O, von Haehling S, Bergmann A, Morgenthaler NG, Anker $\mathrm{SD}$. Mid-region pro-hormone markers for diagnosis and prognosis in acute dyspnea: results from the BACH (Biomarkers in Acute Heart Failure) trial. J Am Coll Cardiol 2010;55:2062-2076.

4. Le Gall JR, Lemeshow S, Saulnier F. A new simplified acute physiology score (SAPS II) based on a European/North American multicenter study. JAMA 1993;270:2957-2963.

5. Vincent JL, Moreno R, Takala J, Willatts S, De Mendonça A, Bruining H, Reinhart CK, Suter PM, Thijs LG. On behalf of the Working Group on Sepsis-related Problems of the European Society of intensive care medicine. Intensive Care Med 1996;22:707-710.

6. Horwich TB, Patel J, MacLellan WR, Fonarow GC. Cardiac troponin I is associated with impaired hemodynamics, progressive left ventricular dysfunction, and increased mortality rates in advanced heart failure. Circulation 2003;108:833-838.

7. Pascual-Figal DA, Manzano-Fernández S, Boronat M, Casas T, Garrido IP, Bonaque JC, Pastor-Perez F, Valdés M, Januzzi JL. Soluble ST2, high-sensitivity troponin T- and N-terminal pro-B-type natriuretic peptide: complementary role for risk stratification in acutely decompensated heart failure. Eur J Heart Fail 2011;13:718-725.

8. Rehman SU, Martinez-Rumayor A, Mueller T, Januzzi JL Jr. Independent and incremental prognostic value of multimarker testing in acute dyspnea: results from the ProBNP Investigation of Dyspnea in the Emergency Department (PRIDE) study. Clin Chim Acta 2008;392:41-45.

9. Maisel AS, Krishnaswamy P, Nowak RM, McCord J, Hollander JE, Duc P, Omland T, Storrow AB, Abraham WT, Wu AH, Clopton P, Steg PG, Westheim A, Knudsen CW, Perez A, Kazanegra R, Herrmann HC, McCullough PA; Breathing Not Properly Multinational Study Investigators. Rapid measurement of B-type natriuretic peptide in the emergency diagnosis of heart failure. N Engl J Med 2002;347:161-167.

10. Cook NR. Use and misuse of the receiver operating characteristic curve in risk prediction. Circulation 2007;115:928-935.

11. Pencina MJ, D'Agostino RB Sr, Steyerberg EW. Extensions of net reclassification improvement calculations to measure usefulness of new biomarkers. Stat Med 2011;30:11-21.

12. Lassus J, Gayat E, Mueller C, Peacock WF, Spinar J, Harjola VP, van Kimmenade R, Pathak A, Mueller T, Disomma S, Metra M, PascualFigal D, Laribi S, Logeart D, Nouira S, Sato N, Potocki M, Parenica J, Collet C, Cohen-Solal A, Januzzi JL Jr, Mebazaa A; GREAT-Network. Incremental value of biomarkers to clinical variables for mortality prediction in acutely decompensated heart failure: the Multinational Observational Cohort on Acute Heart Failure (MOCA) study. Int $J$ Cardiol 2013;168:2186-2194. 